\title{
The Ominous Ouzo Party - A Case Series of Four Patients with Accidental Alkali Ingestion
}

\author{
Marcel Vetter (DD ${ }^{1,2}$ \\ Timo Rath ${ }^{1,2}$ \\ Jürgen Siebler ${ }^{1,2}$ \\ Maximilian Waldner ${ }^{1,2}$ \\ Markus F Neurath ${ }^{1,2}$ \\ Lukas Pfeifer ${ }^{3}$
}

'Department of Medicine I, FriedrichAlexander-Universität ErlangenNürnberg, Erlangen, Germany; ${ }^{2}$ Deutsches Zentrum Immuntherapie, DZI, Erlangen, Germany; ${ }^{3}$ Department of Gastroenterology and Interventional Endoscopy, Krankenhaus Barmherzige Brüder, Regensburg, Germany
Correspondence: Lukas Pfeifer Department of Gastroenterology and Interventional Endoscopy, Krankenhaus Barmherzige Brüder, Prüfeninger Straße

86, Regensburg, Bavaria, 93049, Germany $\mathrm{Tel}+499413692051$

Fax +49 94I3692055

Email Lukas.Pfeifer@barmherzige-

regensburg.de
Background: Ingestion of alkaline fluids is a common problem, which can lead to perforations, strictures and malignancy. We present a rare case series of several patients who accidentally ingested the same alkaline substance in different doses.

Methods: We investigated four patients with accidental ingestion of dishwashing liquid. All patients underwent gastroscopy within $24 \mathrm{~h}$ after inpatient admission. Gastroesophageal lesions were classified according to the Zargar classification for corrosive ingestions.

Results: Esophagogastric lesions were predominantly found at the distal esophagus and the small curvature of the stomach. The severity of these lesions ranged from mild erosions (Zargar 2A) to marked necrosis (Zargar 3A). Our data suggest that the degree of these lesions correlated with the amount of ingested toxin and duration of the inpatient stay. However, a low symptom severity or inconspicuous otolaryngologic examination did not exclude severe gastroesophageal lesions.

Conclusion: Our data suggest that the severity of gastroesophageal lesions correlates with the amount of ingested alkaline substance. Symptom burden and an otolaryngologic examination are not sufficiently predictive for the severity of gastroesophageal lesions. The composition and quantity of the swallowed liquid should be determined.

Keywords: alkali ingestion, gastroscopy, toxin dose, case series

\section{Introduction}

Several people of a local soccer club met for a social evening in their club house. The innkeeper was absent that day but left a key for the group to enter the inn. During cleaning up at the end of the evening, the group discovered two bottles of a dry anise flavoured aperitif ("ouzo"). The opportunity was taken, shot glasses were filled, distributed among the party and toasted. While drinking, four people immediately recognized a nasty unpleasant taste. During the night, one person developed abdominal pain and presented himself in our emergency room. Based on the gastroscopic findings showing a large and deep necrosis in the antrum and erosive lesions in the oesophagus, the other participants of the ouzo party were immediately called in. Further investigation revealed that one bottle contained dishwashing liquid instead of ouzo, which caused alkaline burns in all members that drank from this bottle.

With an estimated global annual incidence of $110 / 100,000$, the ingestion of corrosive substances is a common problem. ${ }^{1}$ Accidental oral ingestion of alkaline substances is mostly found in children. In adults, unlike in our example, such intoxications usually occur with suicidal intent. ${ }^{2}$ Alkaline substances cause 
Table I Clinical and Endoscopic Characteristics of Patients with Alkali Ingestion

\begin{tabular}{|c|c|c|c|c|}
\hline Patients & $\mathbf{P} \mathbf{I}$ & $\mathbf{P 2}$ & $\mathbf{P 3}$ & P4 \\
\hline Age & 34 & 56 & 70 & 38 \\
\hline Sex & M & M & $\mathrm{F}$ & M \\
\hline Volume & I shot glass & $\begin{array}{l}\sim 0.5 \text { shot } \\
\text { glass }\end{array}$ & $\sim 0.25$ shot glass & Very small sip \\
\hline $\begin{array}{l}\text { Duration of inpatient } \\
\text { stay }\end{array}$ & 9d & $5 d$ & $3 d$ & Id \\
\hline Symptoms & $\begin{array}{l}\text { Abdominal } \\
\text { pain }\end{array}$ & $\begin{array}{c}\text { Nausea } \\
\text { Vomiting } \\
\text { Abdominal } \\
\text { pain }\end{array}$ & Burning on the lips, mouth, throat and retrosternal & $\begin{array}{l}\text { Retrosternal } \\
\text { pain }\end{array}$ \\
\hline $\begin{array}{l}\text { Otolaryngological } \\
\text { council }\end{array}$ & $\begin{array}{l}\text { Normal } \\
\text { findings }\end{array}$ & $\begin{array}{l}\text { Normal } \\
\text { findings }\end{array}$ & $\begin{array}{l}\text { Base of tongue and hypopharynx slightly reddened (chemical } \\
\text { burn } I^{\circ} \text { ) }\end{array}$ & Normal findings \\
\hline Zargar classification & $3 A$ & $2 B$ & $2 A-2 B$ & $2 A$ \\
\hline Most distal lesions & Pylorus & Pylorus & Small curvatur & Antrum \\
\hline Initial CRP (mg/l) & 19.4 & 12.8 & 37.1 & 14.3 \\
\hline $\begin{array}{l}\text { Initial Leucocytes (1000/ } \\
\mu L)\end{array}$ & 13.07 & 7.88 & 11.98 & 6.37 \\
\hline
\end{tabular}

colliquation necrosis, resulting in an increased risk of acute perforation. Furthermore, a considerably increased risk for developing strictures and neoplasia exists.

We thus report a rare case series of accidental ingestions in several adult patients with ingestion of different amounts of the same alkaline fluid. This enables the analysis of a dose dependent toxin effect. Moreover, it vividly illustrates the relevance of taking a careful history and considering the possibility of fluid mislabeling, especially in cases with clinical symptoms.

\section{Materials and Methods}

\section{Patient Recruitment}

One patient self-presented to our emergency department. After confirming the diagnosis of alkali ingestion, the other three individuals who had been drinking from the same bottle were phoned and called in for further diagnostics. Each of the contacted individuals presented to our clinic for further diagnostic testing.

\section{Diagnostics}

All patients were hospitalized, given a medical history and a physical examination. The blood sample also included inflammatory parameters. Furthermore, an ENT medical inspection of the oral pharynx and an esophagogastroduodenoscopy (EGD) were performed.

EGD was performed in all patients using high definition white light endoscopes (Olympus Exera III HQ190) within $24 \mathrm{~h}$ after presentation in the hospital. All lesions were classified according to the Zargar classification for corrosive ingestions. ${ }^{3}$ Grade 1 includes only mucosal edema and erythema. Grade 2 is defined by the presence of ulcerations (2A: superficial ulcerations; 2B: deep discrete or circumferential ulcerations), whereas grade 3 is characterized by necrosis (3A: focal necrosis; 3B: extensive necrosis). A perforation defines grade $4 .^{2}$

Twelve weeks later, patients were contacted by telephone to inquire about symptoms of esophageal stricture.

\section{Results}

The four investigated patients were between 34 and 70 years old and three of them were male. None of these patients had known prior gastrointestinal disease.

The host's extraneous history revealed that the ouzo bottle was filled with an alkaline liquid (dishwashing liquid), which contained potassium hydroxide and 
hypochlorite. Patient 1 drank one shot glass, patient 2 approximately half a shot glass, patient 3 approximately a quarter shot glass and patient 4 only sipped a little (Table 1).

All patients suffered from abdominal and/or retrosternal pain. Patient 2 vomited and patient 3 had a burning sensation in the mouth and throat. During EGD, lesions were most pronounced in the distal esophagus (Figure 1, first row). In the stomach, lesions were distributed mainly along the small curvature (Figure 1, second and third row). Lesions were classified according to the Zargar classification and ranged from grade $2 \mathrm{~A}$ to $3 \mathrm{~A}(\mathrm{P} 1: 3 \mathrm{~A}, \mathrm{P} 2$ : $2 \mathrm{~B}, \mathrm{P} 3$ : 2A-2B, P4: 2A). Interestingly, the degree of mucosal damage correlated with the amount of toxin ingested: while the two patients that drank $1 / 4$ of a shot glass or less $(\mathrm{P} 3+\mathrm{P} 4)$ had less pronounced lesions than the patient with half a shot (P2), the patient with a full shot glass (P1) had even necrotic lesions (Figure 2). Additional otolaryngological evaluation by the ENT (ear-nose-throat) department revealed a slight pharyngeal erythema in patient 3 , whereas ENT inspection of the other patients were unremarkable. Thus, otolaryngological evaluation did not correlate with the degree of more distal mucosal damage. Clinical symptoms and serological markers of inflammation were also not able to predict mucosal damage reliably (Table 1). The duration of inpatient stay varied between 1 and 9 days (P1: 9 days, P2: 5 days, P3: 3 days, P4: 1 day) and tended to be longer in patients with more pronounced lesions (Figure 2).

All patients were offered analgesic therapy as needed. In addition, the patients were initially fasting for at least $24 \mathrm{~h}$ (dependent on endoscopic severity) and a cautious diet was established afterwards. In addition, crystalloids (iv) and pantoprazole were applied.

No patient developed subsequent complications (eg, strictures) within the following 12 weeks (telephone interview). Due to an increased risk for oesophageal neoplasia after corrosive ingestion, we recommended annual surveillance gastroscopy beginning 10 years after the event.

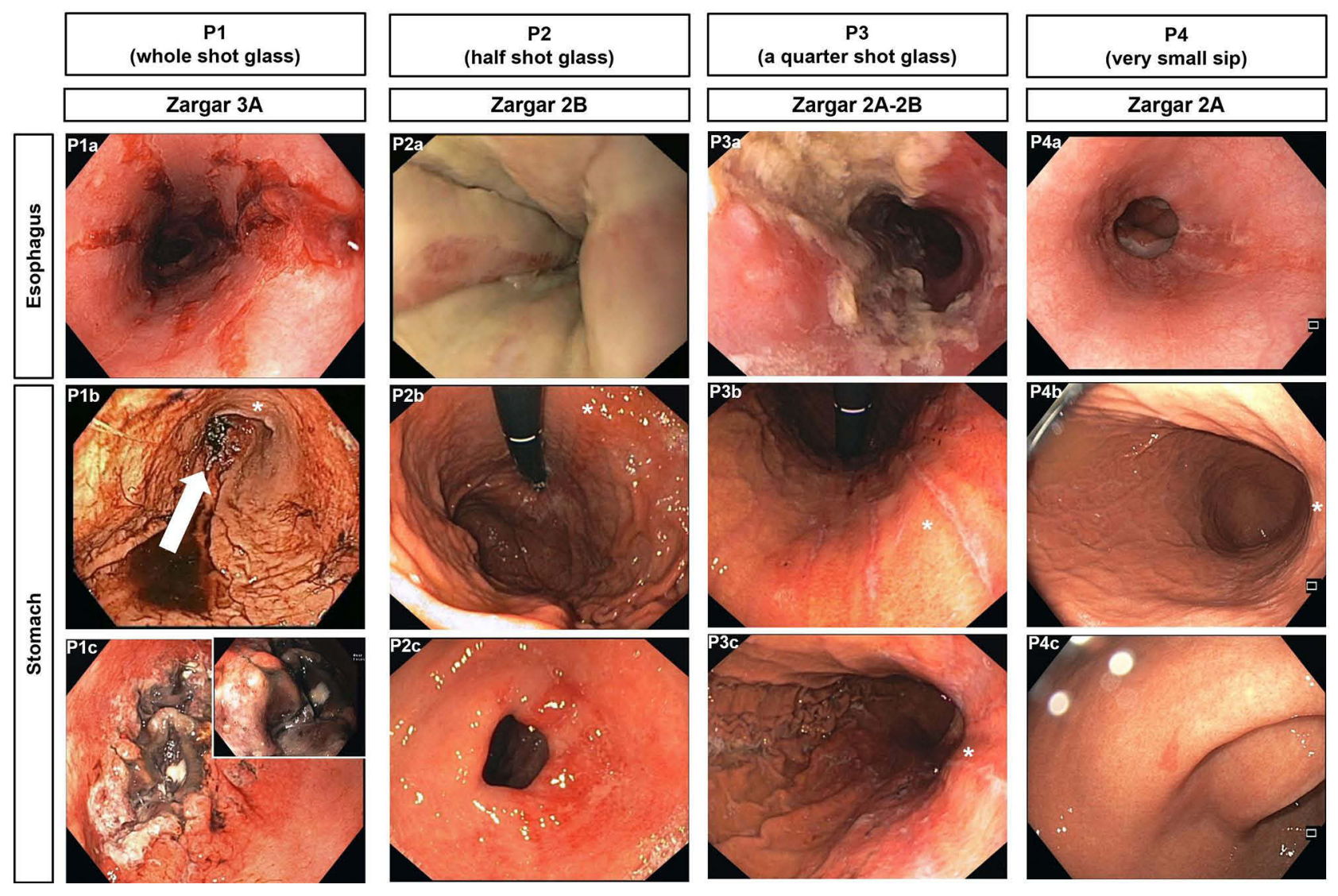

Figure I Images from early performed gastroscopy for patients PI-P4. Esophageal lesions are documented in the first row, the following rows show lesions in the stomach. The white arrow (PIb) marks a focal necrosis (approximately $3 \mathrm{~cm}$ ) in the antrum, which is shown in more detail in PIc (Zargar $3 \mathrm{~A})$. The other patients had ulcerations without necrosis (Zargar 2). The asterisk $(*)$ marks the small curvature. 


\section{Discussion}

We report a rare case series of accidental ingestions in several adult patients with different amounts of the same alkaline fluid.

Interestingly, a pattern in the severity of the gastroscopic findings is apparent, which is probably due to the length of stay and the way of passage through the upper gastrointestinal tract. In the esophagus, the findings were more pronounced distally than proximally. The elongated lesions at the small curvature suggest that the fluid moved along this route towards the antrum.

The particular situation of studying several patients with different doses of the same toxin allowed us to analyze a dose effect. Our data suggest that a higher amount of the toxin cause overall more damage and is probably associated with a longer inpatient stay. In a large monocentric, retrospective study, patients with ingestion of alkaline and acidic substances were examined with regard to risk factors for higher-grade damage in the upper gastrointestinal tract. While a significant influence of the toxine dose was demonstrated for acids, this was not possible for alkaline substances. However, a dose effect also seems plausible for alkaline substances. Perhaps, such an influence could not be observed due to the different $\mathrm{pH}$ values and combinations of substances investigated in this study. ${ }^{4}$ Our data suggest that it is important to elicit the exact toxin dose.

In our patients a low symptom burden, a negative ENT medical report or normative inflammatory parameters in the serum did not rule out a higher degree of damage in the upper gastrointestinal tract. A poor negative predictive value for symptoms is consistent with data from a large study, in which no significant correlations of clinical symptoms and the severity of mucosal damage during EGD were found among 378 children with caustic ingestions. ${ }^{5}$ It is known that in the case of liquid corrosive ingestions, damage is greater gastroesophageal than oral due to a longer residence time. ${ }^{6}$ This explains well the low concordance of oral and gastroesophageal damage in our study.

We observed a tendency for longer inpatient stays in patients with higher grade esophagosgastric damage. According to a retrospective study $(n=179)$ an early gastroscopic assessment of the lesions is the best predictive factor for short-term prognosis. ${ }^{7}$ Index upper gastrointestinal endoscopy should be performed within $24-48 \mathrm{~h}$, as it is assumed that the risk of perforation increases after $48 \mathrm{~h} .^{2,8}$ In a large retrospective multicentre study $(n=21,682)$ the group with an index endoscopy after $48 \mathrm{~h}$ had a worse clinical outcome and a prolonged hospitalization. ${ }^{9}$ However, in cases of supposed perforation, pain, epiglottic swelling or severe burns in the hypopharynx, gastroscopy is contraindicated. ${ }^{2}$

The prevalence for oesophageal neoplasia after corrosive ingestion is assumed to be high and to increase over time (according to an older study: 2-30\% within 10-30 years). ${ }^{2}$ We usually recommend annual surveillance endoscopy beginning 10 years after the event. However, there is a lack of data on whether earlier screening might be beneficially for selected patients (eg, with more pronounced lesions). It sounds reasonable that surveillance should especially focus on the areas of damage in the index examination.

Moreover, this case illustrates very clearly that primary anamnesis or mislabeling can lead on the wrong track and critical questioning especially in cases of unusual taste or symptoms is required.

This study benefits from the analysis of different doses of the identical alkaline liquid, extensive data collection, and a vivid story, but is limited by the small number of patients.

\section{Conclusion}

In summary, we present a rare case series of patients who accidentally ingested the same alkaline substance in different quantities. Our data suggest that higher toxin doses could lead to higher gastroesophageal damage, so the toxin dose should always be attempted to be determined. Even in very mild symptoms, normal serological markers for inflammation and inconspicuous oral findings, higher degrees of gastroesophageal damage can not be excluded. Performing upper gastrointestinal endoscopy within $48 \mathrm{~h}$ after ruling out contraindications appears helpful in assessing the clinical course. Furthermore, this case series shows that false labelling of fluids should be considered in cases of unusual taste or symptoms.

\section{Abbreviations}

EGD, Esophagogastroduodenoscopy; ENT, ear-nosethroat.

\section{Statement of Ethics}

The study protocol conforms to the ethical guidelines of the revised Declaration of Helsinki (2000, Edinburgh) and was approved by the local Ethics Committee at the Friedrich-Alexander University Erlangen-Nürnberg (file number 175_21 Bc). Informed consent for this study and publication of the clinical details were obtained from all patients. 


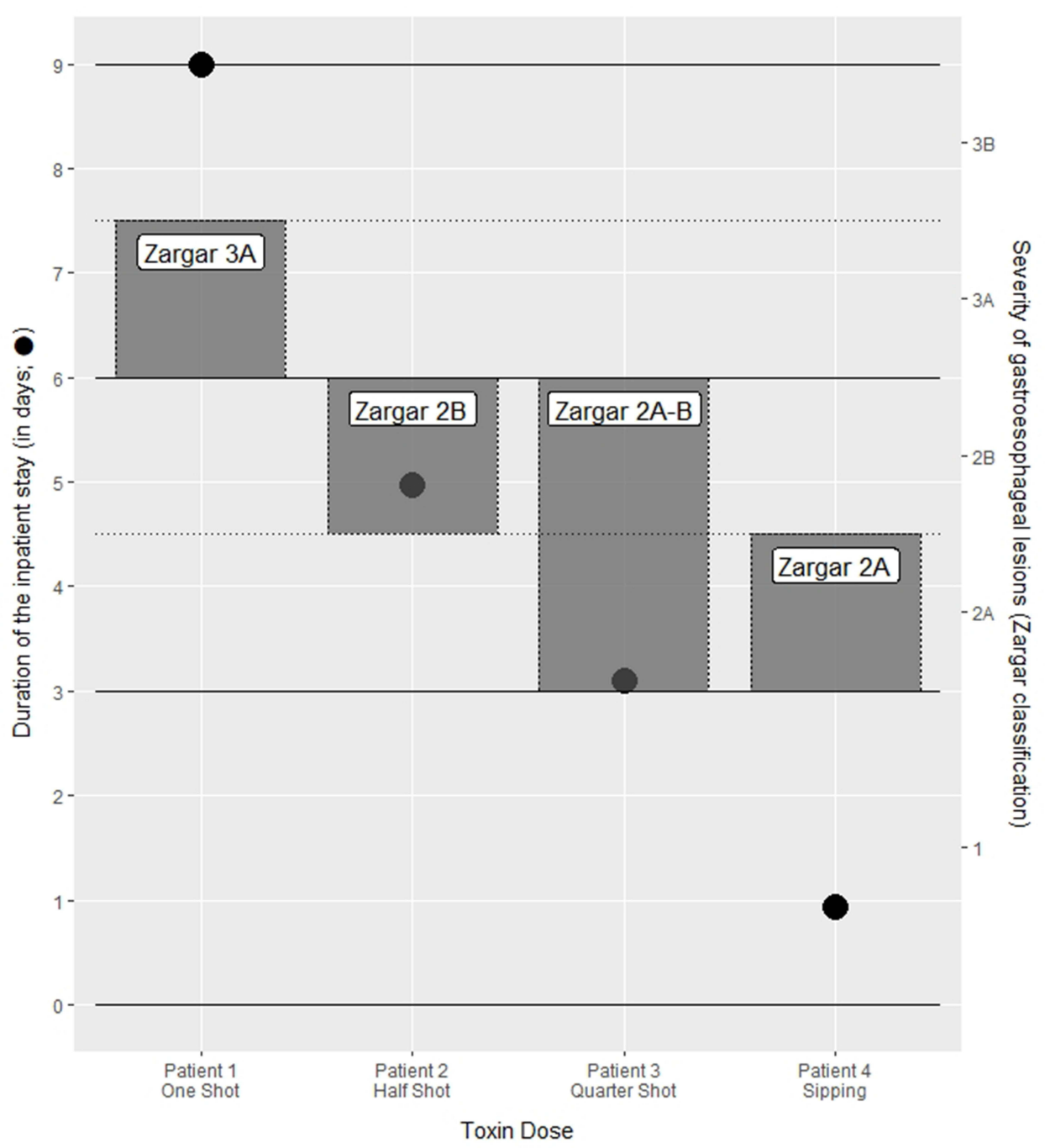

Figure 2 Correlation of the toxin dose with the severity of the gastroesophageal lesions and the duration of the inpatient stay. The black circles ( $\bullet$ ) indicate the duration of the inpatient stays, whereas the rectangles show the corresponding Zargar classification.

\section{Author Contributions}

All authors made substantial contributions to conception and design, acquisition of data, or analysis and interpretation of data; took part in drafting the article or revising it critically for important intellectual content; agreed to submit to the current journal; gave final approval of the version to be published; and agree to be accountable for all aspects of the work.

\section{Funding}

There is no funding to report.

\section{Disclosure}

The authors have no conflicts of interest to declare.

\section{References}

1. Hall AH, Jacquemin D, Henny D, Mathieu L, Josset P, Meyer B. Corrosive substances ingestion: a review. Crit Rev Toxicol. 2019;49 (8):637-669. doi:10.1080/10408444.2019.1707773

2. Contini S. Caustic injury of the upper gastrointestinal tract: a comprehensive review. World J Gastroenterol. 2013;19(25):3918. doi:10.3748/wjg.v19.i25.3918

3. Ali Zargar S, Kochhar R, Mehta S, Kumar mehta S. The role of fiberoptic endoscopy in the management of corrosive ingestion and modified endoscopic classification of burns. Gastrointest Endosc. 1991;37(2):165-169. doi:10.1016/S0016-5107(91)70678-0

4. Chen Y-J, Seak C-J, Kang S-C, et al. A new perspective of the risk of caustic substance ingestion: the outcomes of 468 patients in one North Taiwan medical center within 20 years. Clin Toxicol. 2021;59 (5):409-417. doi:10.1080/15563650.2020.1822998

5. McGuigan A, Chicoine L, Lovejoy H Predictability of Esophageal Injury from Signs and Symptoms: a Study of Caustic Ingestion in 378 Children. Pediatrics. 1983;71(5):767-770. 
6. Lusong MAAD, Timbol ABG, Tuazon DJS. Management of esophageal caustic injury. WJGPT. 2017;8(2):90. doi:10.4292/wjgpt.v8.i2.90

7. Poley J-W, Steyerberg EW, Kuipers EJ, et al. Ingestion of acid and alkaline agents: outcome and prognostic value of early upper endoscopy. Gastrointest Endosc. 2004;60(3):372-377. doi:10.1016/ S0016-5107(04)01722-5

8. Lupa M, Magne J, Guarisco JL, Amedee R. Update on the diagnosis and treatment of caustic ingestion. Ochsner J. 2009;9(2):6.
9. Abbas A, Brar TS, Zori A, Estores DS. Role of early endoscopic evaluation in decreasing morbidity, mortality, and cost after caustic ingestion: a retrospective nationwide database analysis. Dis Esophagus. 2017;30(6):1-11. doi:10.1093/dote/dox010

\section{Publish your work in this journal}

Clinical and Experimental Gastroenterology is an international, peerreviewed, open access, online journal publishing original research, reports, editorials, reviews and commentaries on all aspects of gastroenterology in the clinic and laboratory. This journal is indexed on American Chemical Society's Chemical Abstracts Service (CAS).
The manuscript management system is completely online and includes a very quick and fair peer-review system, which is all easy to use. Visit http://www.dovepress.com/testimonials.php to read real quotes from published authors. 